\title{
The instrumentation of the data acquisition system for the MonRAt telescope
}

M. A. Leigui de Oliveira ${ }^{* 1}$, V. P. Luzio ${ }^{1}$, A. F. Barbosa ${ }^{\dagger 2}$, H. P. Lima Jr ${ }^{2}$, V. A. Ferraz ${ }^{3}$, A. B. $\operatorname{Vilar}^{4}$, M. S. A. B. Leão

1-Universidade Federal do ABC (UFABC), Santo André, Brazil;

2-Centro Brasileiro de Pesquisas Físicas (CBPF), Rio de Janeiro, Brazil;

3-Universidade Federal do Rio de Janeiro (UFRJ), Rio de Janeiro, Brazil;

4-Instituto Federal de Educação, Ciência e Tecnologia (IFRJ), Rio de Janeiro, Brazil;

5-Faculdade Independente do Nordeste (FAINOR), Vitória da Conquista, Brazil.

E-mail: teiquidufabc.edu.br

MonRAt is a compact telescope designed to detect photons generated by ultra-high energy cosmic-ray particles in the atmosphere. The telescope is composed of a 64-pixel multianode photomultiplier tube in the focus of a parabolic mirror. Ultraviolet-passing filters are positioned in front of the photocathode to select photons within the wavelength range of nitrogen fluorescence. The data acquisition system consists of a front-end, a set of preamplifiers and FPGA-based boards able to record trigger times and waveforms from each channel. MonRAt is being designed to detect air fluorescence and Cherenkov radiations under different atmospheric and geomagnetic conditions.

The 34th International Cosmic Ray Conference,

30 July- 6 August, 2015

The Hague, The Netherlands

\footnotetext{
*Speaker.

${ }^{\dagger}$ Deceased.
} 


\section{Introduction}

Cosmic ray showers with energies exceeding $10^{17} \mathrm{eV}$ have long been observed through measurements of atmospheric nitrogen fluorescence light [四] [] [ []]. In these experiments, one measures light intensity profiles in the near ultraviolet band, after the excitations of nitrogen molecules by charged particles from air showers. The large number of particles near the axis of ultra-high energy showers is responsible for producing light with enough intensity to be detected, even by photomultipliers placed kilometers away from the emission point. The absolute fluorescence yield has been measured for some electron energies, air pressures and temperatures [团 [ [వ] [ [G] [0], but further and more precise results are needed. In this work, we describe the data acquisition system for the MonRAt telescope $[\mathbb{[}]$, a compact fluorescence detector with which we intend to precisely characterize the fluorescence yield under several atmospheric conditions.

\section{The apparatus}

MonRAt $^{1}[$ [ $]$ is a portable detector designed to observe fluorescence photons in the atmosphere. The main component of MonRAt is a MultiAnode PhotoMultiplier Tube (MAPMT) with 64 pixels (model H7546B by Hamamatsu [9]), which is positioned at the focus of a parabolic mirror. The signals generated by the MAPMT are then collected, amplified, formatted and digitized by a set of electronic boards, the data acquisition system described here. The digitizers are FPGA-based boards, able to record waveforms (ADCs) and time stamps (TDCs) from each channel and send these informations through a USB interface to a computer. In figure W, we present in a schematic diagram the main parts of the MonRAt experiment [ए]] [ए]]. Part of the electronics has been purchased and part has been developed in our laboratories. In the next sections we will discuss them in detail.

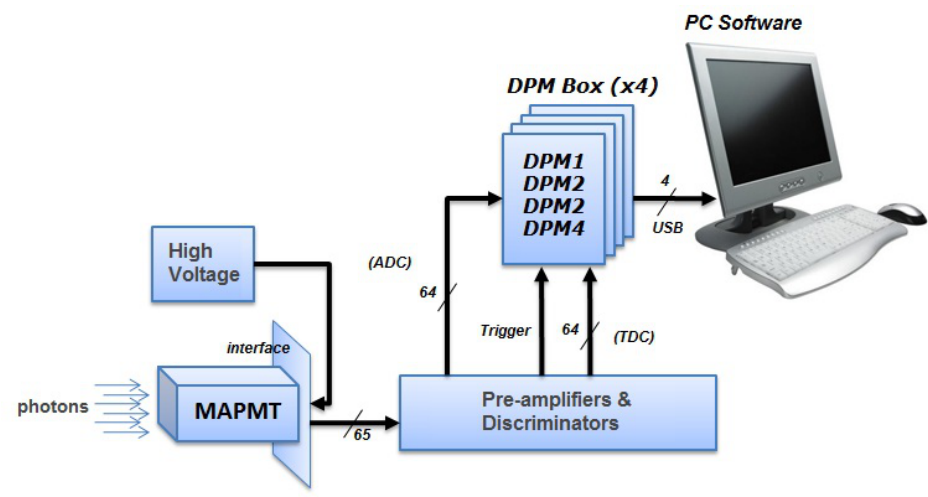

Figure 1: The MonRAt block diagram.

\subsection{MAPMT front-end board and the divider boards}

The signals are collected by a front-end board (model SIB164A, manufactured by Vertilon Corporation [[2]]). It conditions the MAPMT and provides its connectivity through a 76 pin socket

\footnotetext{
${ }^{1}$ For the abbreviation of Atmospheric Radiation Monitor, in Portuguese.
} 


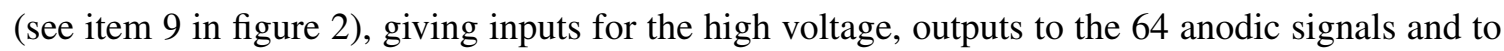
the last dynode signal, as well as a common ground.

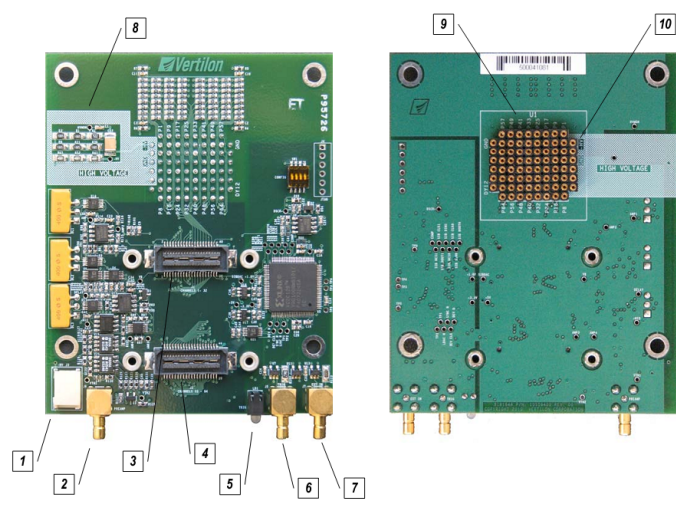

\author{
1- high voltage input; \\ 2- preamplifier output; \\ 3- interface connector J1 (model SBC090P); \\ 4- interface connector J2 (model SBC090P); \\ 5- trigger indicator LED; \\ 6- last (12th) dynode signal; \\ 7- external input; \\ 8- high voltage area; \\ 9- socket to the H7546B MAPMT; \\ 10- MAPMT high voltage input.
}

Figure 2: The front-end board SIB164A by Vertilon [ㅁ] ].

In figure $\square$, there is a picture of the front-end board with a description of its main components. The anodic signals are routed to 2 connectors ( $\mathrm{J} 1$ and $\mathrm{J} 2$ ), located on the backside of the board, which allows us to conveniently mount the system with the photocathode facing the optical arrangement and plug the cables on the other side. The signals have been taken from the front-end board through 2 flat cables (model CNN020), able to collect 32 channels each. Then, they are split by 2 divider boards and sent to another 8 boards, where they will be amplified and formatted, as described in the next section.

\title{
2.2 Preamplifiers and discriminators
}

The MAPMT generates signals with low amplitudes that require amplification. We use circuits with low noise, broadband and performing the functions of integrator, amplifier and filter, employing the operational amplifier OPA657, as shown in figure [3. The time constant of the second stage ( $\tau=R_{\text {shape }} C_{\text {shape }}$ ) sets the rising and falling times for the signals which must be compatible to the sampling times of $\sim 10 \mathrm{~ns}$ of the analog to digital converter. Moreover, they form a low-pass filter that influences the bandwidth of the preamplifier.

The discriminator generates TTL pulses for signals with amplitudes above a given threshold. A fast analog comparator with propagation time of $7 \mathrm{~ns}$, model AD8561 from Analog Devices (see lower part of figure B] $)$, is used. The input voltage $\left(V_{\text {in_disc }}\right)$ is compared to a constant threshold voltage $\left(V_{t h}\right)$, adjusted by the potentiometer R2. The integrated circuit (REF43GS) provides a stable voltage reference of $2.5 \mathrm{~V}$, then a digital output signal, compatible with the TTL standard, is produced by the comparator.

The preamplification and discrimination stage is done in 8 boards of 8 channels each, like the one in figure 6 . Subsequently, the signals are sent to the Data Processing Modules (DPMs).

\subsection{Data processing module}

The data acquisition of the MonRAt detector is performed in Data Processing Modules (DPMs), circuits responsible for digitalization of signals and communication with the computer. The system 

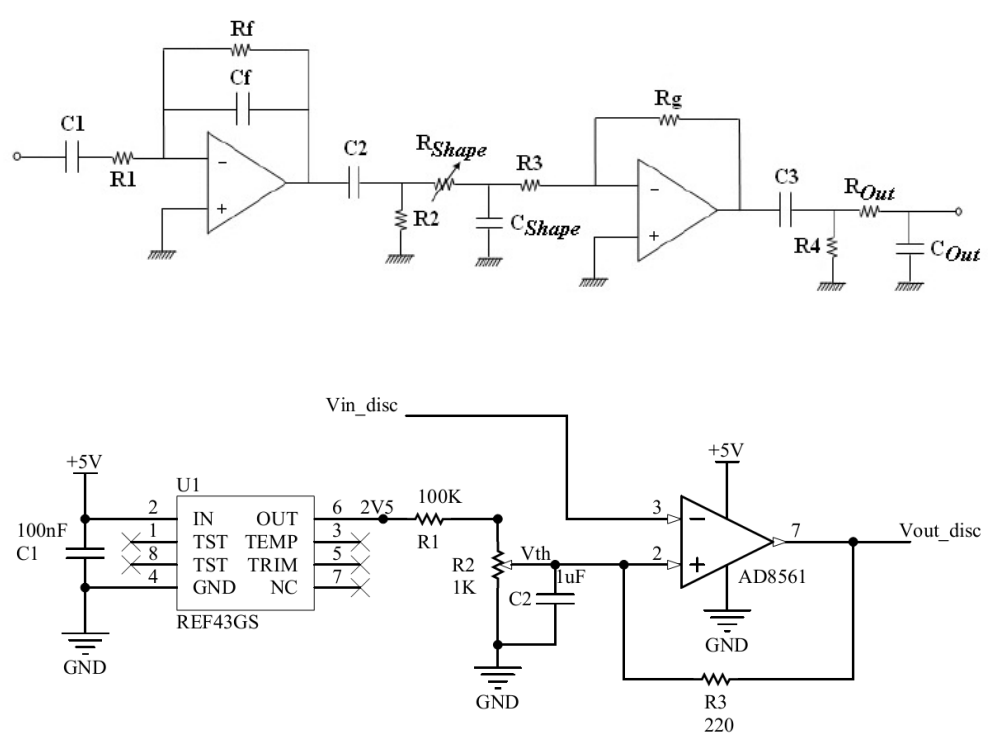

Figure 3: Upper panel: the preamplifier circuit; lower panel: the discriminator circuit.
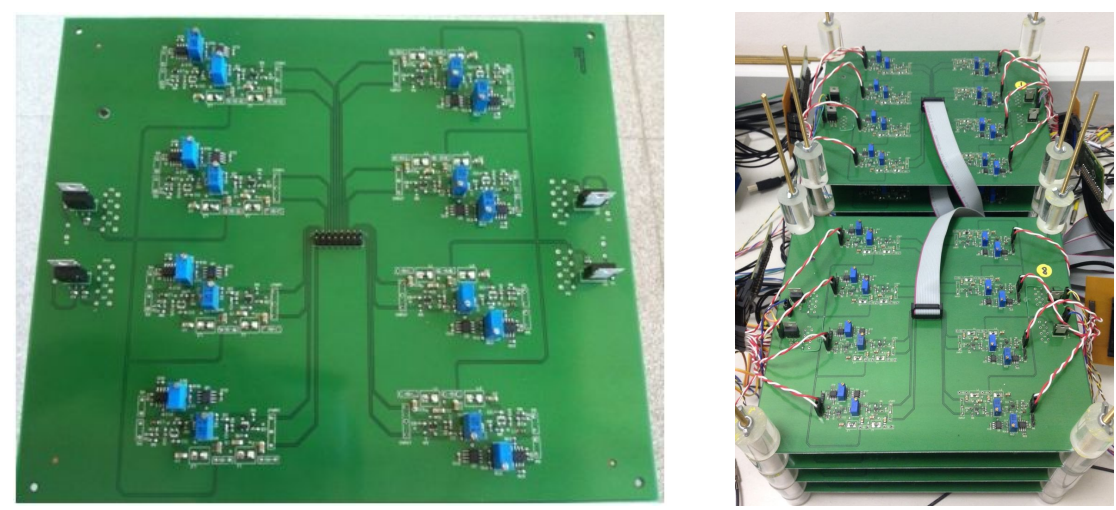

Figure 4: Photographs of 1 board preamplifiers and discriminators (left) and the 8 boards for the whole system (right).

is composed of 16 DPMs, divided in 4 boxes, totalizing 64 analog input channels. Each DPM has a digital input, which triggers the data acquisition process.

The DPM is based on a logic Field Programmable Gate Array (FPGA) device, as shown in figure 5(a). It has 2 ADCs with 2 channels each, 8 channels for time-to-digital conversions, the FPGA device and 24 channels of digital input/output pins and a bus interface for communication with a DB-25 connector. The communication with the computer is done via a USB board interface installed in one DPM, called master, positioned in the top of the box. The communication with the other 3 DPMs in the box, called slave, is done via the DB-25 connector. The connectors for the 4 analog inputs into the DPM board are $50 \Omega$ LEMO supporting high frequency signals.

The two ADCs have sampling rate of $60 \mathrm{MS} / \mathrm{s}$ and resolution of 12 bits, within a dynamic range of $2 \mathrm{~V}_{\mathrm{pp}}$ and theoretical resolution of $2 / 2^{12} \mathrm{~V} \sim 0.5 \mathrm{mV}$. The converted digital word remains available in synchronism with a clock signal, generated by the FPGA, of $60 \mathrm{MHz}$. The first channel data are synchronized with the rising edge of the clock, while data from the second channel are 


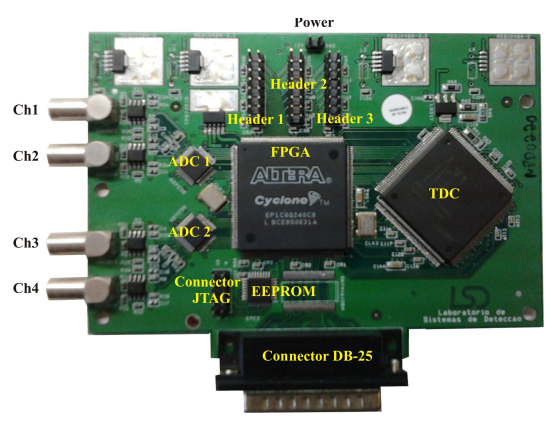

(a) One single board for 4 input channels.

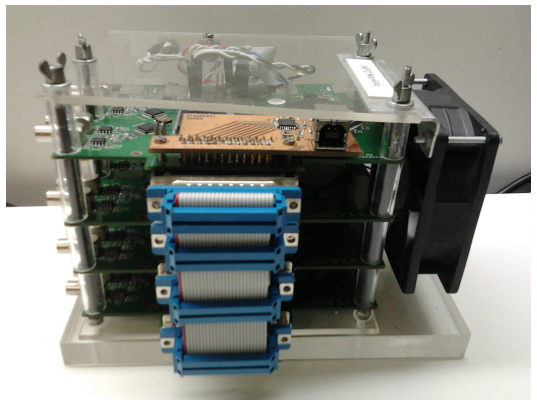

(b) A DPM box, consisting of a set of 4 DPMs (1 master and 3 slaves).

Figure 5: The Data Processing Modules (DPMs).

synchronized with the falling edge. Following the diagram shown in figure $\mathbf{6}$, the data available in the internal memory are transferred by the USB bus communication through the control performed by the FPGA circuits.

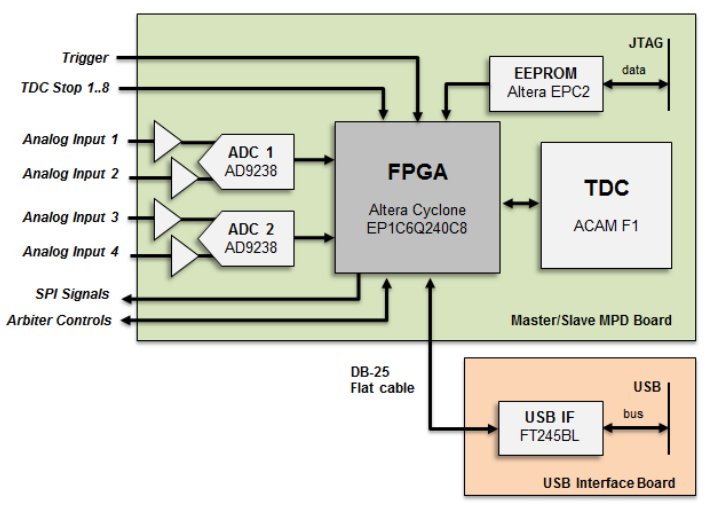

Figure 6: Block diagram of the DPM and the USB communication board.

The DPM has a single Time-integrated Digital Converter (TDC), manufactured by Acam, with 8 channels with resolution of $\sim 120 \mathrm{ps}$ and 16 bit precision. It can measure time intervals between $5 \mathrm{~ns}$ and $7.8 \mu \mathrm{s}$. The resolution is calibrated by a crystal oscillator through a phase locked loop circuit and is adjustable by internal configuration. After the signal is detected, the TDC begins the measurement of time and waits for the stop signals. The converted output value is identified by its conversion channel at the digital bus, connected to the FPGAs. They receive the converted data and send them to the computer via the USB port.

\section{Software}

The data acquisition software is developed using the platform ROOT [ए3] ] and the library of functions for the USB transceiver. The software is written in C++, running under Linux operating system, and is object-oriented. It runs the data acquisitions, proving signal visualizations and output files for offline analyses. The class diagram is shown in figure $\square$. They are related by one 
inheritance (between the DPM and DPMMonRAt classes) and one single dependency (between DPM and ftd2xx classes).

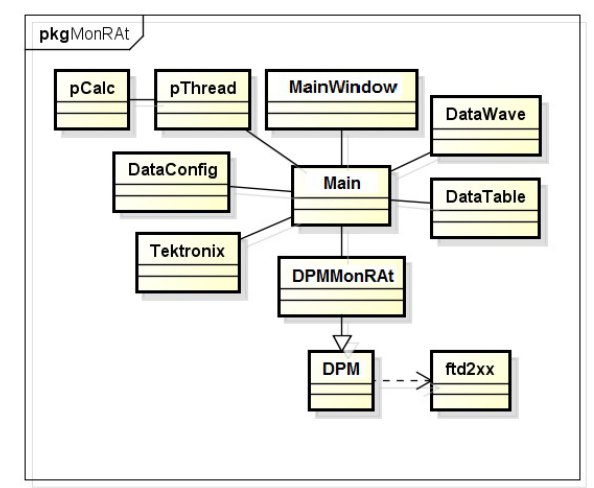

Figure 7: Simplified diagram of classes used in the data acquisition software.

The software features a graphic interface for signal visualizations and user selectable options for output waveforms or amplitude tables into files. Event counters for each device are shown on screen and a status bar displays the conditions of the connections, sending messages to the user.

\section{The single photoelectron and the gain of individual pixels}

We tested MonRAt data acquisition system, measuring of the gain of individual MAPMT channels by using the single photoelectron procedure. A minimal amount of light ( $\sim 1$ photon) has been impinged on each MAPMT pixel, with a blue LED, both placed in a dark room (see figure [8), and read the resulting ADC signals, after preamplification and conversion in the DPMs. Then, we obtained the spectrum of signals of multiplied electrons in the anodes and measured the gain of each MAPMT channel.

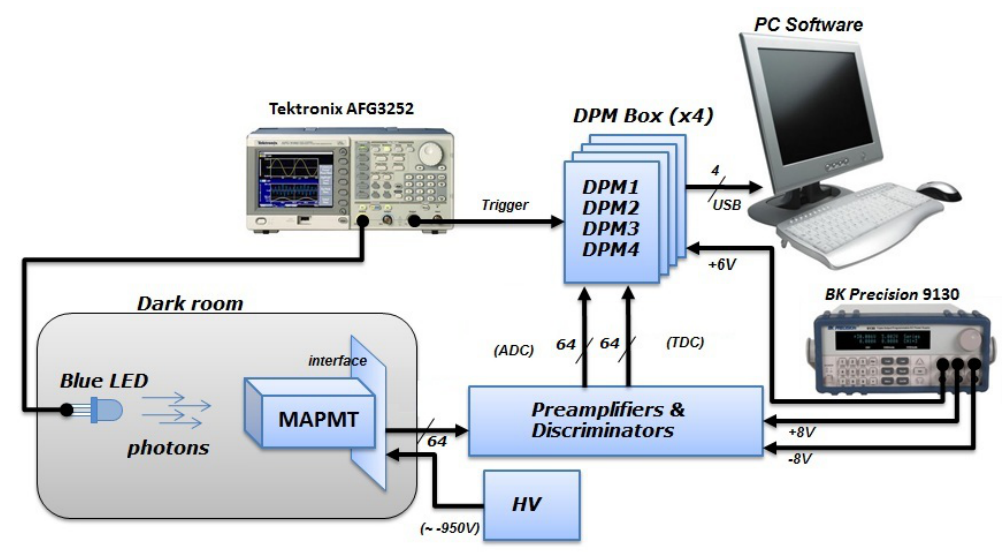

Figure 8: The single photoelectron setup.

In the left side of figure $Q$, we can see the single photoelectron spectrum of a given channel and, on the right side, the histogram of gains for all the channels. We estimate an average gain of $1.78 \times 10^{6}$, in accordance with the manufacturer of the photomultiplier [Q]. 
(a)

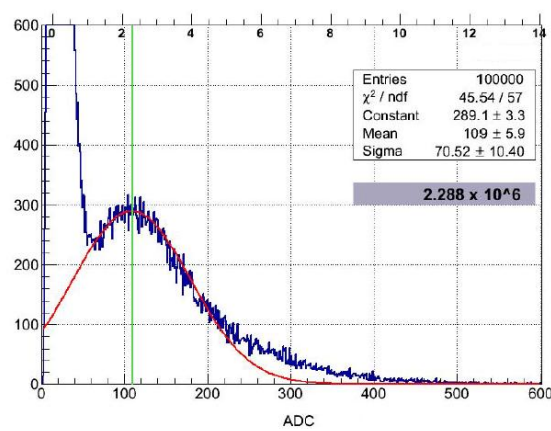

(b)

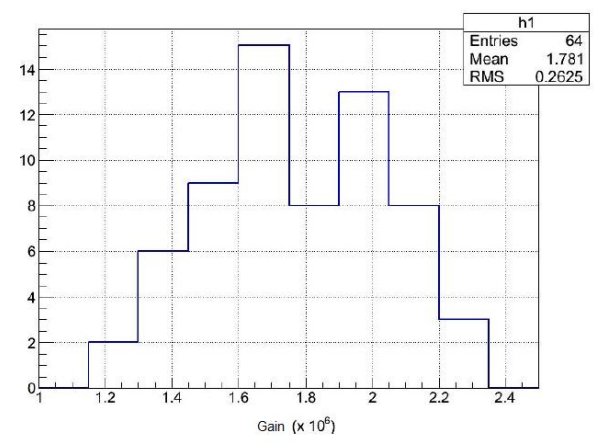

Figure 9: (a) single photon for channel 27; (b) histogram of gains.

\section{The light spot and transit test}

Another test of the MonRAt system was to use the same blue LED as light source in the dark room, aligning it near the optical axis at a distance of $2.5 \mathrm{~m}$ from a small spherical mirror of $15 \mathrm{~cm}$ of diameter. The spot size formed at the MAPMT photocathode can be seen at the upper part of figure 10(a) for an amount of light much larger than actually used during the test. Then, the data acquisition system has been turned on and the signals were read through the MonRAt software. In the lower part of figure 10(a), we plot the histogram of signals: there is one central pixel with counts well above the others (showing the most illuminated pixel) and a small aberration upon two neighbours. This result show us that the MonRAt system was able to collect this small amount of light.
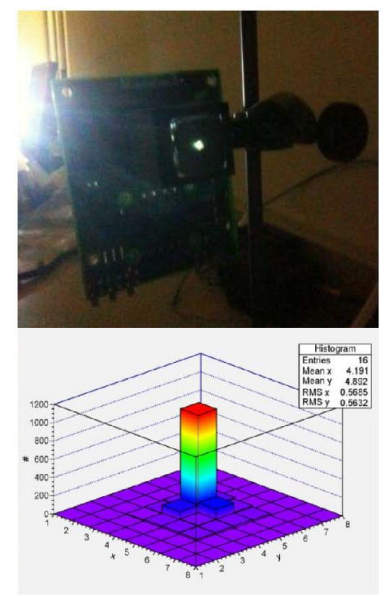

(a) The light spot upon central pixels.

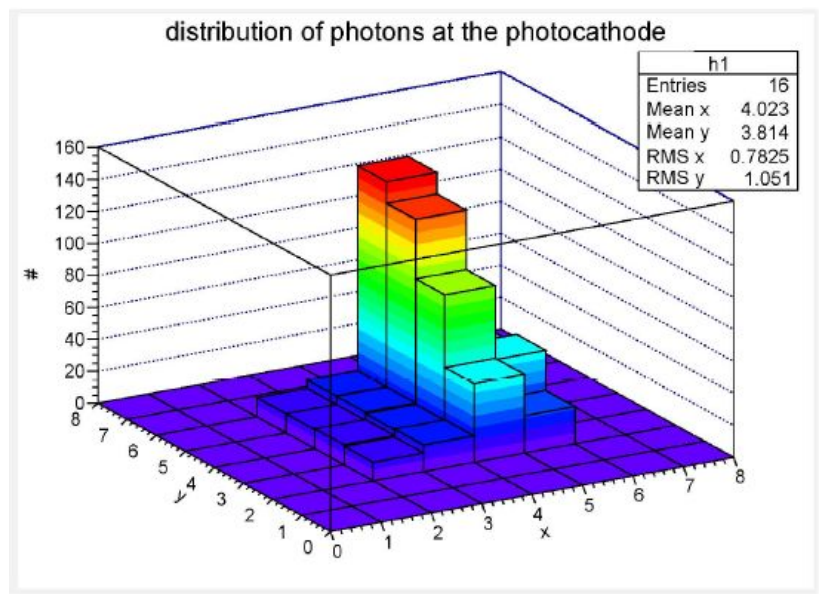

(b) The moving LED test.

Figure 10: Light tests with MonRAt system.

A second test was to progressively displace the LED by some centimeters, simulating a cosmicray shower track moving in the MAPMT field of view. The result is given in figure 10(b). 


\section{Conclusions}

We have presented the design of the MonRAt detector electronics and software, measurements of individual channels gains, and its expected performance under simulated conditions. MonRAt is designed for the detection of atmospheric fluorescence radiation generated by cosmic-ray showers of energies greater than $10^{17.5} \mathrm{eV}$. The detector construction has been finished and the assemblies of the mechanical and optical structures are under way. We soon will be able to measure real events, pioneering detecting the fluorescence radiation in Brazil.

\section{Acknowledgements}

This work has been funded by the Brazilian foundations FAPESP (Fundação de Amparo à Pesquisa do Estado de São Paulo), grant number 2008/00879-4, and CNPq (Conselho Nacional de Desenvolvimento Científico e Tecnológico), grant number 479983/2010-0. The authors are thankful to our colleagues at UFABC and CBPF for the facilities used in the development of this work.

\section{References}

[1] T. Abu-Zayyad et al., Nucl. Instrum. Meth. A, 450 (2000) 253.

[2] Pierre Auger Collaboration. A. Aab et al., accepted for publication in Nucl. Instrum. Meth. (2015), [arXiv: 1502.0132].

[3] M. Fukushima, Prog. Theor. Phys. Supp., 151 (2003) 206.

[4] K. Kakimoto et al., Nucl. Instrum. Meth. A, 372 (1996) 527.

[5] M. Nagano et al., Astropart. Phys., 20 (2003) 293; Astropart. Phys., 22 (2004) 235.

[6] J. W. Beltz et al., Astropart. Phys., 25 (2006) 129.

[7] P. Collin et al., astroph/06121110.

[8] M. A. Leigui de Oliveira et al., Int. J. of Basic \& Applied Sciences, 14 (2014) 32-40.

[9] http://www.hamamatsu.com

[10] V. A. Ferraz, Sistema multicanal para aquisição de dados em um experimento de fluorescência atmosférica, Master Thesis, CBPF (2012), Rio de Janeiro, Brazil.

[11] A. B. Vilar, Instrumentação para observação de fluorescência de raios cósmicos com fotomultiplicadora multianódica, Master Thesis, CBPF (2009), Rio de Janeiro, Brazil.

[12] http://www.vertilon.com

[13] http://root.cern.ch/root 